\title{
Double Trouble: Reducing Gastrointestinal Bleeding due to DAPT Following Acute Coronary Syndromes
}

\author{
Fares Ayoub $^{1} \cdot$ Neil Sengupta $^{1}$
}

Published online: 29 May 2020

(c) Springer Science+Business Media, LLC, part of Springer Nature 2020

Ischemic heart disease is the leading cause of death in the USA [1]. Globally, at least 550,000 new acute coronary syndrome (ACS) events and 200,000 recurrent events occur annually [2]. Antithrombotic therapy is the cornerstone of management of patients with ACS, with dual antiplatelet therapy (DAPT) frequently prescribed for patients undergoing percutaneous coronary intervention (PCI). As a result of the direct pharmacologic effects of DAPT, gastrointestinal bleeding (GIB) occurs as a complication in at least 0.6-3.9\% of patients in the first 30 days following ACS [3]. Aspirin, a member of the family of nonsteroidal anti-inflammatory drugs (NSAIDs), can directly induce peptic ulcer disease by inhibiting cyclooxygenase, decreasing the production of tissue prostaglandins that protect the gastroduodenal mucosa. Thienopyridines such as clopidogrel, ticagrelor, and prasugrel can promote bleeding by impeding the release of platelet-derived growth factors that contribute to angiogenesis and mucosal healing [4]. The combination of aspirin and thienopyridines that constitute DAPT promotes bleeding at de novo or preexisting gastrointestinal lesions caused by aspirin itself, other NSAIDs, or H. pylori infection. Considerably inferior outcomes occur in patients with ACS who develop GIB; in a post hoc analysis of 13,819 patients enrolled in a randomized trial of different antithrombotic regimens following ACS, Nikolsky et al. [5] found GIB to be independently associated with 30-day and 1-year all-cause mortality (hazard ratio [HR] 4.87 and 3.97, respectively). With increasingly potent antithrombotic therapy and estimated one million PCI procedures performed annually in the USA alone, several important questions arise: how common is GIB complicating ACS? More importantly, what factors

Neil Sengupta

nsengupta@medicine.bsd.uchicago.edu

1 Section of Gastroenterology, Hepatology and Nutrition, Department of Medicine, The University of Chicago Medicine, Chicago, IL 60637, USA predict GIB following ACS, and how can GIB be best prevented in this setting?

Several studies have previously attempted to answer these questions. Gaglia et al. identified GIB in $0.7 \%$ of 20,621 patients who underwent $\mathrm{PCI}$ in a 10-year single-center retrospective study. Unadjusted 30-day mortality rate of patients with GIB was $20.5 \%$ compared with $2.4 \%$ of patients without GIB. Older age, shock, acute myocardial infarction, chronic renal insufficiency, and lower baseline hematocrit were independent risk factors for GIB [6]. With remarkably increased mortality rates and limited modifiable risk factors, prevention strategies have focused on the inhibition of gastric acidity and promotion of ulcer healing with proton pump inhibitors (PPI). Table 1 summarizes international societal guidelines regarding PPI use for primary prophylaxis of GIB in patients on DAPT. PPIs reduce the risk of GIB in patients on DAPT; in a nationwide Danish study of 46,301 patients on DAPT, PPI therapy reduced the risk of GIB by $\approx 40 \%$ [7]. The use of PPI for prevention of GIB following ACS has not been straightforward, as several observational and ex vivo studies had previously suggested an interaction between clopidogrel and PPIs where PPIs inhibited the antiplatelet effects of clopidogrel. The Clopidogrel and the Optimization of Gastrointestinal Events Trial (COGENT) [8] was designed to clarify the extent of this interaction using a randomized, double-blind, placebo-controlled study design. In COGENT, rates of GIB were compared between clopidogrel plus omeprazole and clopidogrel alone; the rate of overt upper GIB was reduced with omeprazole as compared with placebo (HR 0.13), and a total of 109 patients had a cardiovascular event, with event rates of $4.9 \%$ with omeprazole and $5.7 \%$ with placebo (HR with omeprazole, 0.99). Ultimately, COGENT provided robust data dispelling many of the concerns regarding the interaction between PPIs and clopidogrel in real-life practice.

In this issue of Digestive Diseases and Sciences, Gupta et al. [9] report the results of their analysis of the Nationwide Readmission Database (NRD) between 2010 and 2014. 
Table 1 Summary of international societal guidelines on proton pump inhibitor (PPI) use for primary prophylaxis of gastrointestinal bleeding (GIB) in patients on dual antiplatelet therapy (DAPT)

\begin{tabular}{|c|c|c|}
\hline Guideline source & Recommendation (strength of recommendation) & Strength of recommendation \\
\hline $\begin{array}{l}2010 \text { ACCF/ACG/AHA Expert Consensus Docu- } \\
\text { ment on the Concomitant Use of Proton Pump } \\
\text { Inhibitors and Thienopyridines }\end{array}$ & $\begin{array}{l}\text { PPIs are recommended to reduce GI bleed- } \\
\text { ing among patients with a history of upper } \\
\text { GI bleeding. PPIs are appropriate in patients } \\
\text { with multiple risk factors for GI bleeding who } \\
\text { require antiplatelet therapy } \\
\text { Routine use of either a PPI or an H2RA is not } \\
\text { recommended for patients at lower risk of } \\
\text { upper GI bleeding, who have much less poten- } \\
\text { tial to benefit from prophylactic therapy } \\
\text { Clinical decisions regarding concomitant use of } \\
\text { PPIs and thienopyridines must balance overall } \\
\text { risks and benefits, considering both CV and GI } \\
\text { complications }\end{array}$ & Not specified \\
\hline $\begin{array}{l}2011 \text { ACCF/AHA/SCAI Guideline for Percuta- } \\
\text { neous Coronary Intervention }\end{array}$ & $\begin{array}{l}\text { PPIs should be used in patients with a history of } \\
\text { prior gastrointestinal (GI) bleeding who require } \\
\text { DAPT } \\
\text { Use of PPIs is reasonable in patients with an } \\
\text { increased risk of GI bleeding (e.g., advanced } \\
\text { age, concomitant use of warfarin, steroids, non- } \\
\text { steroidal anti-inflammatory drugs, Helicobacter } \\
\text { pylori infection) who require DAPT } \\
\text { Routine use of a PPI is not recommended for } \\
\text { patients at low risk of GI bleeding, who have } \\
\text { much less potential to benefit from prophylactic } \\
\text { therapy }\end{array}$ & $\begin{array}{l}\text { Class I, level C evidence } \\
\text { Class IIa, level C evidence } \\
\text { Class III (no benefit), level C evidence }\end{array}$ \\
\hline $\begin{array}{l}2015 \text { ESC Guidelines for the management of } \\
\text { acute coronary syndromes in patients present- } \\
\text { ing without persistent ST segment elevation }\end{array}$ & $\begin{array}{l}\text { Gastric protection with a proton pump inhibitor } \\
\text { is recommended }\end{array}$ & Not specified. \\
\hline $\begin{array}{l}2017 \text { ESC focused update on dual antiplatelet } \\
\text { therapy in coronary artery disease }\end{array}$ & $\begin{array}{l}\text { A PPI in combination with DAPT is recom- } \\
\text { mended }\end{array}$ & Class I recommendation, level B evidence \\
\hline
\end{tabular}

$A C C F$ American College of Cardiology Foundation, ACG The American College of Gastroenterology, AHA The American Heart Association, ESC European Society of Cardiology, SCAI Society for Cardiovascular Angiography and Interventions, PPI proton pump inhibitor, DAPT dual antiplatelet therapy

Their goals were twofold: to identify rates and outcomes of GIB-related readmission in patients who develop ACS at an index hospitalization, and to characterize predictors of readmission in this cohort. Using recommended weighting strategies, they identified 3,520,241 patients who were discharged with ACS. Of those, 25,010 (0.7\%) developed GIB during their index admission and 10,018 (0.3\%) were readmitted with GIB within 30 days. Of those readmitted with GIB, in-hospital mortality was $3.6 \%$, significantly lower than the above rates reported in the literature. Interestingly, the most common etiology of bleeding was gastrointestinal tumors in $17.3 \%$ of readmitted patients, followed by hemorrhoids in $9.8 \%$. Readmitted patients were older and more likely to have comorbid conditions, particularly chronic kidney disease and a history of peptic ulcer disease. The authors also describe resource utilization in this cohort: mean length of stay was 5.8 days and hospital charges for readmitted patients amounted to a remarkable 500 million USD. In a multivariate analysis of patient, hospital, treatment and disease severity-related factors, advanced age, multiple comorbidities, and hemodynamic shock during the index admission were the strongest predictors of readmission within 30 days.

This study has several strengths. First, the use of the NRD guarantees a nationally representative sample. This also ensures large enough patient numbers to facilitate the study of potential risk factors for GIB-related readmission following ACS with sufficient power. Second, the authors present trends for GIB-related readmission between 2010 and 2014, with notable stability of readmission rates across those years. The stability of readmission rates in these years is of particular relevance since they encompass FDA approval and increasing use of the more potent thienopyridines prasugrel (approved in 2009) and ticagrelor (2011) possibly signaling no increase in risk of GIB associated with these medications compared with clopidogrel.

Unfortunately, primarily owing to limitations of the NRD, the current study has several major limitations. First, the lack of adjustment for either antithrombotic medications or PPIs is a significant flaw that limits interpretation of the 
results. The NRD does not contain data on either inpatient or outpatient medications. While the authors assume that most patients admitted for ACS are ultimately treated with DAPT in line with current guidelines, this approach is purely speculative and is unlikely to reflect real-life practice. The lack of medication-related data also confounds interpretation of readmission rates: how many patients were prescribed PPI therapy on their index admission? How many patients already use PPIs for other indications? These questions remain unanswered and are unlikely to be addressed in future studies relying on the NRD.

Second, patients with a history of prior GIB were not excluded by the authors from their readmission analysis. It is well established that patients who develop GIB have an increased risk of readmission compared with controls; a recent analysis of 159,000 hospitalizations for GIB in 2014 showed an overall 30-day readmission rate of $16 \%$ [10]. This leaves open the possibility that some, if not many readmissions included in the analysis, may have been related to rebleeding (rather than new GIB episodes as a complication of PCI). Third, the use of administrative databases such as the NRD for identification of the source of GIB (upper vs. lower) is often problematic due to lack of procedural reports and the poor sensitivity and specificity of ICD-9 codes for GIB. Fourth, although the authors only capture readmissions with the "primary" diagnosis of GIB as listed in the NRD, this approach excludes patients who developed GIB yet were readmitted with another primary diagnosis. These issues cast serious doubts on the accuracy of the authors' estimate of readmission for GIB following ACS, which was their primary outcome.

Efforts at effectively targeting the significant morbidity and mortality associated with GIB complicating ACS will remain limited until larger, unbiased population studies are able to identify intervenable and modifiable risk factors in a population already at a high risk of mortality and evaluate outcomes associated with specific antithrombotic agents, rather than describing readmission rates. Pragmatic efforts may include improving compliance with evidencebased guidelines for PPI prophylaxis in high-risk patients on DAPT through quality improvement initiatives and electronic health record-based reminders, as well as ensuring patients on DAPT are screened and treated for H. pylori infection. Furthermore, providers should stress the importance of avoiding unnecessary NSAIDs and triple antithrombotic-based therapy when feasible. Given the catastrophic implications of GIB in this high-risk population, and multiple avenues already identified for prevention, the time to act is now.

\section{Compliance with Ethical Standards}

Conflict of interest The authors declare that they have no conflict of interest.

\section{References}

1. Murphy SL, Xu J, Kochanek KD, Arias E. Mortality in the United States, 2017 Key findings Data from the National Vital Statistics System. 2017; [cited 2020 May 4]. Available from https://www. cdc.gov/nchs/data/databriefs/db328_tables-508.pdf\#1.

2. Mozaffarian D, Benjamin EJ, Go AS, et al. Heart disease and stroke statistics-2016 update a report from the American Heart Association. Circulation. 2016. https://doi.org/10.1161/ CIR.0000000000000350.

3. Albeiruti R, Chaudhary F, Alqahtani F, Kupec J, Balla S, Alkhouli M. Incidence, predictors, and outcomes of gastrointestinal bleeding in patients admitted with ST-elevation myocardial infarction. Am J Cardiol. 2019. https://doi.org/10.1016/j.amjca rd.2019.05.008.

4. Vaduganathan M, Bhatt DL. Gastrointestinal bleeding with oral anticoagulation: understanding the scope of the problem. Clin Gastroenterol Hepatol. 2017. https://doi.org/10.1016/j. cgh.2016.12.033.

5. Nikolsky E, Stone GW, Kirtane AJ, et al. Gastrointestinal bleeding in patients with acute coronary syndromes: incidence, predictors, and clinical implications. Analysis from the ACUITY (Acute Catheterization and Urgent Intervention Triage Strategy) trial. J Am Coll Cardiol. 2009. https://doi.org/10.1016/j. jacc.2009.07.019.

6. Gaglia MA, Torguson R, Gonzalez MA, et al. Correlates and consequences of gastrointestinal bleeding complicating percutaneous coronary intervention. Am J Cardiol. 2010. https://doi. org/10.1016/j. amjcard.2010.06.011.

7. Sehested TSG, Carlson N, Hansen PW, et al. Reduced risk of gastrointestinal bleeding associated with proton pump inhibitor therapy in patients treated with dual antiplatelet therapy after myocardial infarction. Eur Heart J.. 2019. https://doi.org/10.1093/ eurheartj/ehz104.

8. Bhatt DL, Cryer BL, Contant CF, et al. Clopidogrel with or without omeprazole in coronary artery disease. N Engl J Med. 2010. https://doi.org/10.1056/NEJMoa1007964.

9. Gupta K, Khan A, Manish K, Sawalha K, Abozenah M, Singhania R. Readmissions rates after Myocardial Infarction for Gastrointestinal bleeding: a national perspective. Dig Dis Sci. (Epub ahead of print). https://doi.org/10.1007/s10620-020-06315-1.

10. Siddique SM, Mehta SJ, Lewis JD, Neuman MD, Werner RM. Rates of hospital readmission among medicare beneficiaries with gastrointestinal bleeding vary based on etiology and comorbidities. Clin Gastroenterol Hepatol. 2019. https://doi.org/10.1016/j. cgh.2018.04.039.

Publisher's Note Springer Nature remains neutral with regard to jurisdictional claims in published maps and institutional affiliations. 